\title{
Genome evolution in an ancient bacteria-ant symbiosis: Parallel gene loss among Blochmannia spanning the origin of the ant tribe Camponotini
}

Laura E Williams, Jennifer J Wernegreen

Stable associations between bacterial endosymbionts and insect hosts provide opportunities to explore genome evolution in the context of established mutualisms and assess the roles of selection and genetic drift across host lineages and habitats. Blochmannia, obligate endosymbionts of ants of the tribe Camponotini, have coevolved with their ant hosts for $\sim 40 \mathrm{MY}$. To investigate early events in Blochmannia genome evolution across this ant host tribe, we sequenced Blochmannia from two divergent host lineages, Colobopsis obliquus and Polyrhachis turneri, and compared them with four published genomes from Blochmannia of Camponotus sensu stricto. Reconstructed gene content of the last common ancestor (LCA) of these six Blochmannia genomes is reduced (690 protein coding genes), consistent with rapid gene loss soon after establishment of the symbiosis. Differential gene loss among Blochmannia lineages has affected cellular functions and metabolic pathways, including DNA replication and repair, vitamin biosynthesis and membrane proteins. Blochmannia of $P$. turneri (i.e., $B$. turneri) encodes an intact DnaA chromosomal replication initiation protein, demonstrating that loss of $d n a A$ was not essential for establishment of the symbiosis. Based on gene content, $B$. obliquus and $B$. turneri are unable to provision hosts with riboflavin. Of the six sequenced Blochmannia, $B$. obliquus is the earliest diverging lineage (i.e., the sister group of other Blochmannia sampled) and encodes the fewest protein-coding genes and the most pseudogenes. We identified 55 genes involved in parallel gene loss, including glutamine synthetase, which may participate in nitrogen recycling. Pathways for biosynthesis of coenzyme A, terpenoids and riboflavin were lost in multiple lineages, suggesting relaxed selection on the pathway after inactivation of one component. Analysis of Illumina read datasets did not detect evidence of plasmids encoding missing functions, nor the presence of coresident symbionts other than Wolbachia. Although gene order is strictly conserved in four Blochmannia of Camponotus sensu stricto, comparisons with deeply divergent lineages revealed inversions in eight genomic regions, indicating ongoing recombination despite ancestral loss of recA. In sum, the addition of two Blochmannia genomes of divergent host lineages enables reconstruction of early events in evolution of this 
symbiosis and suggests that Blochmannia lineages may experience distinct, hostassociated selective pressures. Understanding how evolutionary forces shape genome reduction in this system may help to clarify forces driving gene loss in other bacteria, including intracellular pathogens. 
2 Laura E. Williams ${ }^{1,2}$ and Jennifer J. Wernegreen ${ }^{1,3, *}$

3

$4 \quad{ }^{1}$ Duke Center for Genomic and Computational Biology, Duke University, Durham, NC

$5 \quad{ }^{2}$ Current address: Department of Biology, Providence College, Providence, RI

$6 \quad{ }^{3}$ Nicholas School of the Environment, Duke University, Durham, NC

7 * Corresponding author: Jennifer J. Wernegreen, 101 Science Drive, Box 3382, Durham, NC, 27708,

8 919-681-0331, j.wernegreen@duke.edu 
Introduction

The evolution of stable mutualisms between bacteria and insects has occurred many times and

11 involves phylogenetically diverse lineages (Moran, McCutcheon \& Nakabachi, 2008; Moya et al.,

12 2008; Kikuchi, 2009; Douglas, 2014). In many of these symbioses, the limited diets of insect hosts are

13 supplemented by an intracellular bacterial partner. Variation in dietary requirements among insect

14 hosts likely results in differing selective pressure on endosymbiont genes, which in turn impacts

15 endosymbiont genome evolution. For example, Buchnera aphidicola supplement the carbohydrate-rich

16 plant sap diet of aphids with amino acids (Shigenobu et al., 2000), whereas Wigglesworthia species

17 supplement the blood diet of tsetse flies with vitamins and other cofactors (Akman et al., 2002). By

18 contrast, cockroaches and ants of the tribe Camponotini are generally considered omnivores with

19 complex diets. Their bacterial partners, Blattabacterium and Blochmannia, respectively, synthesize

20 essential amino acids and participate in nitrogen recycling (Gil et al., 2003; Lopez-Sanchez et al., 2009;

21 Sabree, Kambhampati \& Moran, 2009). Nitrogen recycling in Blattabacterium occurs via urease and

22 glutamate dehydrogenase, whereas in Blochmannia, it occurs via urease and glutamine synthetase,

23 although the gene encoding the latter enzyme is missing in some Blochmannia species (Williams \&

24 Wernegreen, 2010). In the case of Blochmannia, its nutritional role may be most important at particular

25 stages of the host's lifecycle (Zientz et al., 2006; Feldhaar et al., 2007; Stoll et al., 2010).

26 Genomes of established endosymbionts like Buchnera, Wigglesworthia and Blochmannia are

27 typically characterized by high AT content, elevated mutation rates and extreme stability of gene order

28 (Moran, McCutcheon \& Nakabachi, 2008). Recombination-related genes are often lost during

29 endosymbiont evolution, and very few recombination events are evident in most obligate

30 endosymbiont lineages (Sloan \& Moran, 2013). Genome reduction in established endosymbionts

31 occurs via degradation and loss of individual genes. Gene loss is likely shaped by both relaxed

32 selective pressure due to the stable intracellular niche and genetic drift due to small effective 
33 population sizes and bottlenecks during vertical transmission of symbionts (Andersson \& Kurland, 34 1998;Moran, McCutcheon \& Nakabachi, 2008). The balance between these forces may shift over time

35 for a given association, from the initial acquisition event through the ongoing evolution of the 36 symbiosis. endosymbionts of ants of the tribe Camponotini (Sauer et al., 2000). Blochmannia are closely related to free-living Enterobacteriaceae such as Escherichia coli and form a clade with other obligate endosymbionts including Baumannia, Sodalis and Wigglesworthia (Herbeck, Degnan \& Wernegreen, 2005; Husnik, Chrudimsky \& Hypsa, 2011). The presence of Blochmannia in multiple extant ant genera of Camponotini points to a single colonization event in the ancestral lineage as the origin of the symbiosis (Sameshima et al., 1999; Wernegreen et al., 2009). Based on phylogenetic evidence, the ancestor of Blochmannia may have been a facultative symbiont of insects (Herbeck, Degnan \& Wernegreen, 2005; Wernegreen et al., 2009).

The ant hosts of the four previously sequenced Blochmannia (Camponotus chromaiodes, $C$. floridanus, C. pennsylvanicus and C. vafer) belong to Camponotus sensu stricto. Whereas these host species span the origin of Camponotus $\sim 16-20 \mathrm{MY}$, the association between Blochmannia and the tribe Camponotini is at least twice that old, on the order of $40 \mathrm{MY}$. To reconstruct earlier events in the evolution of this symbiosis, we sequenced the genomes of Blochmannia from two divergent lineages in the tribe Camponotini: the genus Polyrhachis and the Colobopsis lineage.

Though Colobopsis is formally considered a subgenus of Camponotus, phylogenetic analysis of seven nuclear gene fragments revealed it is a separate lineage from Camponotus (Brady et al., 2006;

Moreau \& Bell, 2013). These studies demonstrated that Colobopsis diverged early in the evolution of 6 the tribe Camponotini, and results are consistent with Colobopsis being the sister group of all other 
57 Camponotini sampled, although relationships in this part of the tree were difficult to resolve 58 conclusively. Subsequent work has further suggested that Colobopsis is likely the sister group of all 59 other extant camponotines (pers. comm., P. S. Ward, 2014). In contrast to Camponotus species with 60 published Blochmannia genomes, C. obliquus lives in small twigs and branches, often in the canopy.

61 Polyrhachis diverged later than Colobopsis and is found in the Old World. The deep evolutionary

62 divergence of these host lineages, as well as differences in their geographic range and habitats, provide

63 a valuable opportunity to investigate the evolutionary trajectories of Blochmannia across the

64 Camponotini and to clarify ancient events that shaped this $40 \mathrm{MY}$ old ant-bacterial partnership.

65

66 Materials and Methods

67 Preparation of genomic DNA

A single colony of C. obliquus was collected near Morehead City, North Carolina, USA by B.

69 Guénard, and P. turneri was collected near Townsville, Australia by S. K. A. Robson (see

70 Acknowledgments). Voucher specimens were deposited in the Bohart Museum of Entomology,

71 University of California, Davis (UCDC), corresponding to voucher ID numbers CASENT0221021 (C.

72 obliquus) and CASENT0220426 (P. turneri). We used the Qiagen DNeasy Blood and Tissue Kit to

73 prepare genomic DNA from a pooled sample of seven eggs, two larvae, five pupae, eight minor

74 workers, six major workers and five female alates for $C$. obliquus and a pooled sample of three worker

75 gasters for P. turneri.

77 Sequencing and assembly of Blochmannia genomes

C. obliquus gDNA was sequenced on an Illumina HiSeq to generate 100 bp paired end reads.

79 We modified filter_reads.py (https://github.com/nickloman/xbase/blob/master/short-read-

80 assembly/filter_reads.py) for Sanger FASTQ format and used the script to remove paired reads with 
81 any bases of quality score $<30$, which retained $6,184,892$ read pairs. We assembled this filtered read

82 dataset using Velvet v1.2.07 (Zerbino \& Birney, 2008) with a hash length of 61, exp_cov 200,

83 cov_cutoff 20 and scaffolding turned off. This generated 566 contigs, one of which aligned to $B$.

84 pennsylvanicus using MAUVE (Darling, Mau \& Perna, 2010). We observed an overlap of $116 \mathrm{bp}$

85 between the contig ends, which suggested that de novo assembly had produced a closed genome. To

86 test this, we used Mosaik (Lee et al., 2014) to align the filtered read dataset against a 1260 bp sequence

87 encompassing the joined contig ends and flanking regions. This alignment produced no zero coverage

88 regions, which confirmed that the single contig was the closed B. obliquus genome.

To finish the genome, we used a two-step process applying different alignment programs to

90 confirm the majority genotype, or the base represented by the majority of reads at each position. In the

91 first step, we used Mosaik, which allows the user to set the stringency of mismatch tolerance. We

92 aligned the filtered read dataset against the closed genome sequence with a maximum mismatch

93 threshold of 12 and then removed duplicate read pairs with the DupSnoop module. Using Consed

94 (Gordon, Abajian \& Green, 1998), we generated a questionable consensus bases report and a highly

95 discrepant indels report for the resulting alignment, which did not identify any positions that needed

96 editing.

97 The second step in our finishing process invokes the IndelRealigner module of the Genome

98 Analysis Toolkit (GATK) to ensure accurate identification of indels (DePristo et al., 2011). We aligned

99 the filtered read dataset against the closed genome sequence using BWA (Li \& Durbin, 2009) and then

100 processed the resulting alignment with RealignerTargetCreator and IndelRealigner from the GATK

101 package. We removed duplicate reads using Picard MarkDuplicates

102 (http://broadinstitute.github.io/picard). Finally, we analyzed the processed alignment using VarScan

103 (Koboldt et al., 2009) to identify positions at which the base in the closed genome sequence differed

104 from the majority of aligned reads. We did not identify any such positions that needed editing, 
105 confirming that the single contig was the closed, finished B. obliquus genome. Genome coverage for 106 this alignment averaged 558x. This sequence is deposited in GenBank as accession number CP010049.

107 The Illumina read dataset is deposited in the NCBI Short Read Archive (SRA) as SRP050154.

108 P. turneri gDNA was sequenced on an Illumina Genome Analyzer II (GAIIx) to generate 150

109 bp paired end reads. We used DynamicTrim.pl and LengthSort.pl from the SolexaQA package (Cox,

110 Peterson \& Biggs, 2010) to generate trimmed reads of at least 80 bp with quality score $>30$ for each

111 base. The resulting trimmed read dataset included 9,590,066 read pairs. We assembled this filtered read

112 dataset using Velvet with a hash length of 41, exp_cov 200, cov_cutoff 20 and scaffolding turned off.

113 This generated 8,275 contigs, four of which aligned to B. pennsylvanicus using MAUVE. To close the

114 four gaps, we generated Sanger sequencing reads and used Phred/Phrap/Consed to assemble and

115 manually examine the sequence.

116 To finish the B. turneri genome, we followed the two-step process described above for $B$.

117 obliquus. In the first step, we corrected nine positions based on alignment of the full read dataset

118 against the closed genome sequence using Mosaik. In the second step, we aligned the full read dataset

119 against the corrected sequence using BWA followed by processing with GATK. We analyzed the

120 processed alignment with VarScan and corrected a single position. Genome coverage for this

121 alignment averaged 1223x. The closed, finished genome sequence is deposited in GenBank as

122 accession number CP010048. The Illumina read dataset is deposited in the NCBI Short Read Archive

123 (SRA) as SRP050161.

\section{Annotation of Blochmannia genomes}

126 We used an annotation engine hosted by the Institute for Genome Sciences (IGS) at the

127 University of Maryland School of Medicine to generate an automated annotation of each genome

128 sequence (Galens et al., 2011), which we then manually curated within the MANATEE framework 
129 (http://manatee.sourceforge.net/igs/). Protein-coding genes predicted by the annotation engine were

130 removed if they lacked a Blast-Extend-Repraze (BER) alignment score $<10^{-5}$ to a protein-coding gene

131 from outside of Blochmannia. We manually examined possible frameshifted genes flagged by the

132 annotation engine. For genes with frameshifts in homopolymer tracts, we included the likely position

133 of the frameshift in the GenBank annotation. Because the frameshifts may be corrected by polymerase

134 slippage (Tamas et al., 2008; Wernegreen, Kauppinen \& Degnan, 2010), we consider these to be intact 135 genes.

136 We curated start sites using BER alignments to Blochmannia and closely related species. When 137 possible, we used the gene name and symbol listed in SwissProt for the homologous gene in E. coli to 138 maintain consistency with existing proteobacterial annotations. For conserved hypothetical proteins or 139 proteins with similarity to a protein family but not a specific family member, we did not assign a gene 140 name and refer to them using the locus tag (for example, BTURN675_020).

141 After curating the annotations, we analyzed intergenic regions in each genome with RFAM 142 (Burge et al., 2013) and BLASTX (Altschul et al., 1990) to identify uncalled genes and pseudogenes.

143 In both B. obliquus and B. turneri, RFAM identified three RNA-coding genes ( $f f s, r n p B$ and tmRNA).

144 To identify protein-coding genes, we aligned intergenic regions to the GenBank non-redundant

145 database using BLASTX with the low complexity filter turned off. We manually examined hits with e-

146 value $<10^{-5}$. Pseudogenes were identified by multiple nonsense mutations, frameshifts and/or gaps.

147 Pseudogenes that aligned to intact homologs with $>60 \%$ coverage had at least two nonsense mutations.

148 We annotated pseudogene coordinates using the boundaries of the BLASTX alignments. Analysis of $B$.

149 obliquus intergenic regions detected two genes (cyoD and $s d h D)$ and 15 pseudogenes (dnaA, engD,

$150 \operatorname{gln} A, p d x A, p d x B, p d x J, \operatorname{rib} A, \operatorname{ribB}, \operatorname{ribC}, \operatorname{rib} D, \sec D, \sec F, \operatorname{top} A, y i g B$ and $u v r D)$. Analysis of $B$.

151 turneri intergenic regions detected five genes (cyoD, infA, rpmJ, ycaR and yidD), one frameshifted

152 gene $(y b e Y)$ and one pseudogene of a hypothetical protein (BTURN675_514). 


\section{Phylogenetic analysis}

We classified genes into six MultiFun categories (cell processes, cell structure, information transfer, metabolism, regulation, and transport) by searching for the gene name in the EcoCyc

157 database. Some genes are assigned to more than one MultiFun category, and we included all categories

158 listed for each gene. If the gene had no associated MultiFun terms in EcoCyc or if it had no gene name, 159 such as BPEN_040, we considered the gene unclassified.

160 To construct a phylogeny, we chose Baumannia (NC_007984), Hamiltonella (NC_012751) and

161 Sodalis (NC_007712) as outgroups. We identified orthologs of Blochmannia genes in these genomes 162 using the Reciprocal Smallest Distance (RSD) algorithm (Wall, Fraser \& Hirsh, 2003) with default 163 values for divergence $(0.8)$ and e-value $\left(10^{-5}\right)$. For each of the six MultiFun categories, we randomly 164 selected five genes present in all taxa. There were no duplicates in the resulting set of 30 genes. We 165 excluded $B$. chromaiodes from the phylogeny because its genome sequence is $98.0 \%$ identical to that 166 of B. pennsylvanicus (Williams \& Wernegreen, 2013). We used TranslatorX (Abascal, Zardoya \& 167 Telford, 2010) and MAFFT (Katoh et al., 2005) to construct a multiple sequence alignment for each 168 gene, which we then trimmed with ZORRO (Wu, Chatterji \& Eisen, 2012). We concatenated the 169 trimmed amino acid alignments and used MrBayes v3.2.1 (Ronquist \& Huelsenbeck, 2003) to 170 construct a majority rule consensus tree. To test whether maximum likelihood methods produced the 171 same topology, we analyzed the same dataset using MEGA v6.0 (Tamura et al., 2013) with a $172 \mathrm{cpREV}+\mathrm{G}+\mathrm{I}+\mathrm{F}$ amino acid substitution model, which had the lowest Bayesian Information Criterion 173 (BIC) score, and assessed branch support using 500 bootstrap replicates.

\section{GC skew and DnaA box motif search}


177 et al., 2009) to construct plots of GC skew with $500 \mathrm{bp}$ window size and $50 \mathrm{bp}$ step size. We used

178 Pattern Locator (Mrazek \& Xie, 2006) to search these three genome sequences for the consensus DnaA

179 box motif TTWTNCACA.

180

181 BLAST analysis

182 Because we prepared genomic DNA from whole ants or gasters, the Illumina read datasets

183 include coverage of genomes other than Blochmannia, such as the ant host nuclear and mitochondrial

184 genomes. To determine if the de novo assemblies included contigs from potential Blochmannia

185 plasmids or other bacterial symbionts, we used BLASTN to align all $\geq 500$ bp contigs to the GenBank

186 non-redundant database, limited to bacteria (taxid 2). For contigs with at least one hit of $>30 \%$

187 coverage and an evalue of $<10^{-5}$, we aligned each contig against the full non-redundant database and

188 examined the top hits.

189 To test whether the Illumina read datasets contain evidence of riboflavin biosynthesis genes, we

190 constructed BLAST databases of the C. obliquus and P. turneri read datasets. For C. obliquus, we built

191 a BLAST database using the unaligned reads file generated by Mosaik during the initial finishing step

192 (see above), thereby excluding most reads originating from the rib pseudogenes within the B. obliquus

193 genome. We compiled a set of query genes representing orthologs of conspicuously absent genes,

194 including ribABDEF from B. pennsylvanicus (NC_007292), Baumannia (NC_007984) and E. coli

195 MG1655 (NC_000913), ribH from B. pennsylvanicus and Baumannia, ribC from E. coli and the

196 Camponotus floridanus gene for EF-1alpha-F2 (EFN72500). For analysis of C. obliquus reads, we also

197 included $r i b F$ and $r i b$ pseudogenes from B. obliquus in the query set. We used BLASTN to align the

198 query genes against both BLAST databases and examined the output for alignments with e-values $<10^{-}$ 1995.

Certain gene distribution patterns may be explained by either parallel gene loss or acquisition 
201 via horizontal gene transfer. To test the hypothesis of horizontal gene transfer, we aligned each of the

20255 genes with such patterns against the GenBank non-redundant database using BLAST. We first used

203 BLASTN, and if this search did not return significant hits, we used BLASTX. We examined the 204 taxonomic assignments of the top hits to identify genes with high scoring alignments to bacteria 205 outside of the Enterobacteriaceae, which may support the hypothesis of horizontal gene transfer.

207 Results

208 B. obliquus has the fewest protein-coding genes and most pseudogenes

209 The size and GC content of the B. obliquus and B. turneri genomes are within the ranges

210 observed for Blochmannia of Camponotus sensu stricto (Table 1). Although B. obliquus is on the upper

211 end of the size range, it has the fewest protein-coding genes of the six sequenced Blochmannia. We

212 also detected 15 pseudogenes in B. obliquus, which is an unusually high number for Blochmannia

213 (Table S1). In both B. obliquus and B. turneri, we identified genes that have frameshifts in

214 homopolymer tracts but otherwise are expected to encode a full-length protein. We consider these

215 genes to be functional, because they may be corrected by polymerase slippage during transcription and 216 expressed as full-length proteins (Tamas et al., 2008; Wernegreen, Kauppinen \& Degnan, 2010).

\section{B. obliquus is the earliest diverging Blochmannia lineage sequenced}

To determine the evolutionary relationships of the Blochmannia lineages, we constructed a phylogeny of 30 randomly selected protein-coding genes shared among Blochmannia genomes and the 221 outgroups Baumannia, Hamiltonella and Sodalis. Using Bayesian methods, the resulting phylogeny

222 has $100 \%$ posterior probability at all nodes (Fig. 1). Maximum likelihood methods produced the same 223 topology with $\geq 99 \%$ bootstrap values. In this phylogeny, B. turneri and Blochmannia of Camponotus 224 sensu stricto are more closely related to each other than to B. obliquus. This topology is congruent with 
225 recent phylogenies of the ant host taxa that show Colobopsis as a separate lineage rather than a 226 subgenus of Camponotus (Brady et al., 2006). Hereafter, we use the phrase "Blochmannia of 227 Camponotus" to mean Blochmannia of Camponotus sensu stricto, which does not include Colobopsis.

\section{Assemblies show evidence of Wolbachia but no Blochmannia plasmids or other bacterial}

230 symbionts

231 Because we prepared genomic DNA from whole ants or gasters, the Illumina read datasets

232 include coverage of genomes other than Blochmannia. To determine whether the assemblies generated 233 by Velvet included contigs from other symbionts or possible plasmids, we aligned all contigs $\geq 500 \mathrm{bp}$ 234 against the GenBank non-redundant database using BLASTN. We limited our first search to bacteria 235 (taxid 2). For contigs with hits of evalue $<10^{-5}$ and $>30 \%$ query coverage, we aligned them against the 236 full database. The top hits from this search are shown in Table S2. Analysis of the $C$. obliquus assembly identified 15 contigs with BLAST hits that met the 238 criteria outlined above. Three of these are from the ant nuclear genome and one is from the ant 239 mitochondrial genome (Table S2). The best-scoring hits for the remaining 11 contigs are all matches to 240 Wolbachia, an endosymbiont found in many insect species (Werren, Baldo \& Clark, 2008), including 241 ants (Russell et al., 2012). We did not detect evidence of any other symbiont or any Blochmannia 242 plasmids. Analysis of the $P$. turneri assembly identified eight contigs with BLAST hits that met the 244 criteria outlined above. Four of these are from the ant nuclear genome and three are from the ant 245 mitochondrial genome (Table S2). The best-scoring hit for the remaining contig is to $B$.

246 pennsylvanicus. To examine this further, we aligned the contig to the $B$. turneri genome sequence, 247 which produced a much better alignment with $100 \%$ coverage and $85 \%$ identity. The contig spans the $2483^{\prime}$ end of $\operatorname{prfB}$, a 19 bp intergenic region and the 5 ' end of lys $S$. Coverage of this contig averages $54 \mathrm{x}$, 
249 whereas coverage of the same region in the B. turneri genome sequence averages $1380 \mathrm{x}$. We aligned

250 the full read dataset against both the B. turneri genome sequence and the contig and then did a de novo

251 assembly using only the mapped reads. The resulting assembly did not reconstruct the contig,

252 suggesting that it was an artifact of assembly rather than evidence of a plasmid or other symbiont.

254 Gene content of reconstructed Last Common Ancestor is highly conserved in divergent

255 Blochmannia lineages

256 Using the six sequenced Blochmannia genomes, we reconstructed the likely gene content of the 257 Last Common Ancestor (LCA) of these lineages. Horizontal gene transfer (HGT) is considered 258 unlikely in Blochmannia due to the isolated intracellular niche of these bacteria. Conservation of gene 259 order in Blochmannia genomes supports the hypothesis that HGT is very rare. Additionally, we used 260 BLAST to identify the best hits for genes with distribution patterns consistent with either independent 261 loss in multiple lineages or acquisition via HGT, and we did not find evidence of HGT (see below). For 262 these reasons, we expect very little, if any, gain of genes in Blochmannia lineages, and our 263 reconstruction of the LCA includes any intact gene found in at least one of the sequenced genomes. By this definition, the genome of the LCA consists of 690 genes (Fig. 2). Most of these genes 265 are retained in the Blochmannia genomes, with 568 genes, or $82 \%$ of the gene content of the LCA, 266 found in all six species. The complete ortholog table can be found as Table S3. Our reconstruction may 267 underestimate LCA gene content, because it is possible that genes were lost independently from all 268 lineages at some point after divergence from the LCA. For example, B. chromaiodes, $B$. 269 pennsylvanicus and B. obliquus encode a pseudogene of $u v r D$, but no sequenced Blochmannia has an 270 intact $u v r D$. This is the only Blochmannia gene found solely as a pseudogene. This gene may have 271 been intact and functional in the LCA, but it is not represented in our reconstruction here. Sequencing 272 of additional taxa, including Blochmannia from other deeply divergent ant host genera such as 
273 Opisthopsis, will further refine reconstruction of the ancestral lineage.

275 Gene content differences among divergent Blochmannia lineages include important cellular 276 functions

277 DNA replication and repair

278 The dnaA chromosomal replication initiation protein is intact in B. turneri, detectable as a 279 pseudogene in B. obliquus and missing in all four sequenced Blochmannia of Camponotus (Fig. 2). In 280 other gamma-proteobacteria, DnaA initiates replication by binding to 9-bp sequences called DnaA 281 boxes within the origin of replication (Zakrzewska-Czerwinska et al., 2007). GC skew analysis predicts 282 that the origin of replication is adjacent to $m n m G$ in most Blochmannia species (Fig. 3), so we searched 283 the intergenic regions flanking $m n m G$ in B. obliquus, B. pennsylvanicus and B. turneri for the 284 consensus DnaA box motif TTWTNCACA (Schaper \& Messer, 1995).

285 We found two matches in B. turneri, none in B. obliquus and one match in B. pennsylvanicus.

286 By comparison, Buchnera aphidicola, which retained dnaA, has two DnaA boxes in oriC (Mackiewicz 287 et al., 2004); therefore, it is possible that DnaA can initiate replication in B. turneri with only two 288 consensus DnaA boxes. However, the presence of a DnaA box in B. pennsylvanicus, which lacks dnaA, 289 suggests that these motifs are not necessarily associated with DnaA function in Blochmannia. In fact, 290 when we searched the entire length of each genome, we found multiple matches to the consensus DnaA 291 box motif, which are unlikely to be involved in DnaA function as described for gamma-proteobacteria 292 such as E. coli (Hansen et al., 2006). More work is needed to understand how the differential 293 distribution of DnaA in Blochmannia species affects control of DNA replication and whether DnaA 294 boxes play a role in replication initiation in B. turneri.

295 Loss of DNA repair mechanisms is a common characteristic of obligate intracellular symbionts 296 of insects. In the six sequenced Blochmannia, two genes involved in base excision repair, mutM and 
$m u t Y$, are differentially distributed. B. obliquus encodes $m u t M$ but is missing $m u t Y$, whereas $B$. turneri

298 and the four Blochmannia of Camponotus are missing mutM and encode mutY (Fig. 2). Both protein

299 products act on 8-oxoG, which can mispair with adenine (Michaels et al., 1992). MutM excises 8-oxoG

300 when it is paired with cytosine, thereby initiating base excision repair. If 8-oxoG is not removed prior

301 to replication, MutY removes the mispaired adenine, enabling repair. Inactivation of either gene leads

302 to an increase in GC-to-TA transversions, which may contribute to the reduced genomic GC content

303 observed in Blochmannia and other insect endosymbionts (Lind \& Andersson, 2008).

$\underline{\text { Vitamin biosynthesis }}$

Pyridoxal 5'-phosphate (PLP), the catalytically active form of vitamin B6, is an important

enzyme cofactor. With very few exceptions, insects and other animals cannot synthesize vitamin B6

308 (Tanaka, Tateno \& Gojobori, 2005). Biosynthesis of PLP in E. coli and other gamma-proteobacteria

309 occurs via a pathway encoded by seven genes (Mukherjee et al., 2011). This pathway is conserved in

B. turneri and Blochmannia of Camponotus, with the exception of epd/gapB, which is missing from all

311 six sequenced Blochmannia (Fig. 4). The function of epd may be fulfilled by gapA, which is present in

312 all six species and can compensate epd mutants in E. coli (Yang et al., 1998). In B. obliquus, only $d x s$

313 and $\operatorname{ser} C$ are conserved, whereas $p d x A B H J$ are missing. We detected pseudogenes of three of these

314 genes. Based on these gene losses, B. obliquus appears unable to provision its ant host with vitamin B6.

Riboflavin (vitamin B2) biosynthesis is another vitamin synthesis pathway with differential

316 gene distribution in these Blochmannia lineages. Riboflavin is essential for synthesis of the cofactors

317 flavin mononucleotide (FMN) and flavin adenine dinucleotide (FAD) (Abbas \& Sibirny, 2011).

318 Blochmannia of Camponotus encode five riboflavin biosynthesis genes ( $\mathrm{rib} A B C D H$ ), which comprise

319 the complete pathway in E. coli. By contrast, these genes are missing in both B. obliquus and $B$.

320 turneri. We detected pseudogenes of ribABCD, but not ribH, in $B$. obliquus. The ribF gene, which 
321 encodes an enzyme that synthesizes FMN and FAD from riboflavin, is conserved in all six sequenced 322 Blochmannia species.

323 It is possible that riboflavin biosynthesis genes are encoded on a plasmid, by a secondary

324 symbiont (Lamelas et al., 2011), or even within the ant host nuclear genome (Husnik et al., 2013). As

325 discussed above, we detected Wolbachia but no plasmids or other symbionts in the assemblies. To

326 investigate possible alternative sources of riboflavin, we analyzed the Illumina read datasets. Using

327 BLASTN, we aligned a query set of rib genes from B. pennsylvanicus, Baumannia and E. coli against

328 the reads. We also included the elongation factor alpha F2 (EF-1alpha-F2) gene from Camponotus

329 floridanus in the query set to assess representation of the ant host nuclear genome in the read datasets.

330 Because B. obliquus has pseudogenes of four riboflavin biosynthesis genes, we used the subset of reads

331 that did not align to the B. obliquus genome to build the C. obliquus BLAST database.

We found no evidence of intact riboflavin biosynthesis genes in the read datasets. For reads

333 from $P$. turneri gasters, we detected no BLASTN hits with e-values $<10^{-5}$ to the query riboflavin

334 biosynthesis genes. For reads from C. obliquus ants, some reads aligned to the B. obliquus

335 pseudogenes. Based on examination of the BLAST alignments, these reads likely originated from the

336 pseudogenes, but they have more mismatches than permitted by Mosaik and were included in the

337 unaligned reads file. When we considered only reads that did not align by BLASTN to the rib

338 pseudogenes, we detected only three reads aligning to the query riboflavin biosynthesis genes with

339 evalues $<10^{-5}$. A single read, but not its mate, aligned to ribE from B. pennsylvanicus with only $58 \%$

340 coverage and an evalue of $\sim 10^{-6}$, and a single read pair aligned to $r i b A$ from E. coli with $100 \%$ identity

341 and $100 \%$ coverage, which may be due to contamination or presence of gut-associated bacteria. These

342 single reads contrast with the 558x coverage of the B. obliquus genome. For both read datasets,

343 multiple reads aligned to the $C$. floridanus EF-1alpha gene with evalues $<10^{-20}$, confirming that the

344 reads include coverage of the host nuclear genome. Our analysis of the Illumina read datasets did not 
345 reveal how these symbiotic systems compensate for the loss of riboflavin biosynthesis in the

346 Blochmannia partners.

$348 \underline{\text { Membrane proteins }}$

All six sequenced Blochmannia encode tolA, an inner membrane protein and a component of the Tol-Pal system; however, there are structural differences among the species. In E. coli, TolA has

351 three domains: a transmembrane domain that anchors the protein in the cytoplasmic membrane, a 352 central periplasmic domain with high alpha-helix structure and a globular periplasmic domain 353 (Godlewska et al., 2009). In Blochmannia, five of the six species have a stretch of 80-100 bp tandem 354 repeats in tolA, which varies in length and repeat sequence among species (Table 2). By contrast, $B$. 355 obliquus shows no evidence of this repeat region, which is also not found in the $E$. coli homolog. E. 356 coli tolA has a repeat region, but it occurs in a distinct location in the protein and consists of shorter 357 repeats (Zhou et al., 2012). In addition, the length of B. obliquus tolA (771 bp) is shorter than tolA in 358 the other Blochmannia species (990-1305 bp) and E. coli (1266 bp).

The function of tolA in an insect endosymbiont such as Blochmannia is unknown. In E. coli, the

360 Tol-Pal system is thought to interact with phage particles and colicins (Godlewska et al., 2009). Some 361 structural features described for E. coli TolA are conserved in Blochmannia, such as the N-terminal 362 transmembrane domain. Both the TMHMM server v 2.0 and Phobius predicted one transmembrane 363 helix within the first 50 amino acids of TolA from each sequenced Blochmannia genome. Six of the 364 seven genes comprising the two Tol-Pal operons (ybgC-tolQ-tolR-tolA and tolB-pal-ybgF) in E. coli 365 are conserved in all six sequenced Blochmannia, with only $y b g C$ missing. The Tol-Pal system may be 366 important in Blochmannia for host-endosymbiont interactions. 
We placed gene losses in a phylogenetic context to identify parallel losses (Fig. 5), which we define as independent loss of the same gene in multiple lineages separated by a lineage that retained the gene. By this definition, we identified 55 genes that were subject to parallel loss in these Blochmannia lineages. An alternative explanation for the distribution patterns of these 55 genes is acquisition by horizontal gene transfer. To test this explanation, we aligned each of the 55 genes to the GenBank nonredundant database using BLAST and identified only two genes ( $i c d$ and yqiC) for which the top hits were to bacteria outside of the Enterobacteriaceae. The top BLASTN hit for icd was to Candidatus Profftella, a beta-proteobacterial endosymbiont of the Asian citrus psyllid. BLASTN did not return any significant hits for $y q i C$, but the top BLASTX hit was to Vibrio litoralis. Although it is possible that these genes were acquired by Blochmannia via horizontal gene transfer, for the purposes of our analysis we consider parallel gene loss a more likely explanation for their distribution patterns. initiation protein $d n a A$, the DNA mismatch repair protein $m u t S$ and DNA topoisomerases topA and topB. Three of the 15 genes ( $a c n B, g l t A$ and $i c d$ ) encode enzymes of the tricarboxylic acid (TCA) cycle and complete this pathway in B. turneri. By contrast, B. obliquus and Blochmannia of Camponotus retained only TCA genes involved in energy generation. In general, parallel loss of these 15 unique $B$. turneri genes in B. obliquus and Blochmannia of Camponotus may indicate differing selective pressures on B. turneri and its ant host. sequenced Blochmannia. Glutamine synthetase was independently lost in both B. vafer and B. obliquus. In other Blochmannia, this enzyme may play an important role in nitrogen recycling for the 
393

394

395

396

ant host (Feldhaar et al., 2007). Parallel loss of $g \ln A$ suggests that this enzyme is not essential to the symbiosis and its function can be fulfilled by an alternative pathway, such as arginine biosynthesis via carbamoyl phosphate synthase (Williams \& Wernegreen, 2010). A few parallel loss events involved genes from the same metabolic pathway, such as coaADE and $d f p$ (coenzyme A biosynthesis), ispADFGH (terpenoid backbone biosynthesis) and ribABCDH (riboflavin biosynthesis). Loss of these genes may reflect relaxed selection on components of a pathway after inactivation of a gene within that pathway.

In addition to genes involved in metabolism, genes encoding outer membrane and secretory proteins, including $\operatorname{omp} A$, $\operatorname{opr} C, \sec B D F$ and $\operatorname{ton} B$, were lost independently in multiple lineages, possibly affecting communication between endosymbiont and host cells. Finally, parallel loss of five hypothetical proteins with unknown function emphasizes the need to better characterize these proteins and understand their contributions to the symbiosis. Maintenance of these hypothetical proteins in particular lineages suggests that they are under selective pressure specific to host lineages.

\section{Blochmannia lineages experienced multiple inversions}

Although gene order is conserved among the four sequenced Blochmannia of Camponotus, comparisons including B. obliquus and B. turneri revealed inversions in eight genomic regions involving between two and 34 genes (Figs. 3 and 6). Two of these regions (labeled D and G in Figs. 3 and 6) show evidence of multiple separate inversion events (Table S3), suggesting that they may be inversion "hotspots". To determine which lineage likely experienced inversion events, we compared gene order in the eight regions to that of close relatives strain HS (CP006569), which is a member of a

\section{Sodalis-allied clade, and Baumannia cicadellinicola str. Hc (NC_007984). For some regions, these} close relatives provide information on the probable gene order of the ancestral Blochmannia lineage.

\footnotetext{
Based on these comparisons, we hypothesize that two inversions occurred in the lineage leading to $B$.
} 
417 obliquus (labeled A and B), one occurred in the lineage leading to B. turneri (labeled F), one occurred

418 in the ancestral lineage of B.turneri and Blochmannia of Camponotus (labeled C), and one occurred in

419 the ancestral lineage of the four sequenced Blochmannia of Camponotus (labeled E). For the remaining

420 three regions, our comparisons were inconclusive.

\section{Discussion}

Comparative genomics of Blochmannia lineages spanning the origin of the ant tribe

424 Camponotini allows us to reconstruct early events in the evolution of this symbiosis. Previous

phylogenetic analyses identified a clade of secondary endosymbionts of mealybugs as the closest

426 relatives of Blochmannia, suggesting Blochmannia may have originated from an ancestor of this group

427 (Wernegreen et al., 2009). Reconstruction of the last common ancestor of the Blochmannia analyzed

428 here showed most genes in the LCA (82\%) have been retained in all six Blochmannia species

429 sequenced to date. This is consistent with the hypothesis that the ancestor was an endosymbiont with

430 an already reduced genome. Alternatively, Blochmannia may have undergone a similar trajectory as

431 that proposed for Blattabacterium, which may have originated as a free-living associate that

432 experienced substantial and rapid gene loss after acquiring an endosymbiotic lifestyle but before

433 diverging into extant lineages (Patino-Navarrette et al., 2013). Sequencing the genomes of

434 Blochmannia of other deeply divergent lineages, such as Opisthopsis, and closely related lineages

435 outside of Blochmannia may provide data to distinguish these two hypotheses.

436 We found that gene content differences among Blochmannia lineages involve several key

437 functions, including information transfer, metabolism, and cell-cell communication, which may affect

438 the functioning of this mutualism across ant host lineages. Regarding metabolism, the biosynthesis

439 pathways for two vitamins, riboflavin and vitamin B6, vary among the sequenced Blochmannia

440 lineages. Plants and most bacteria encode a riboflavin biosynthesis pathway, but animals lack this 
441 pathway. Many insect endosymbionts, such as Baumannia, Blattabacterium, Buchnera, Hamiltonella,

442 Sodalis and Wigglesworthia, synthesize riboflavin. Young aphids depend on the supply of riboflavin

443 from Buchnera for growth and development (Nakabachi \& Ishikawa, 1999). Our analyses showed that

444 B. obliquus and B. turneri have both lost the ability to synthesize riboflavin, whereas Blochmannia of

445 Camponotus have retained this pathway and can provision the ants with this vitamin. These differences

446 in riboflavin biosynthetic functionality illustrate how the nutritional roles of Blochmannia in this

447 mutualism change during co-evolution with hosts.

448 In other endosymbiont systems, loss of riboflavin biosynthesis is compensated by either a

449 secondary symbiont or transfer of the genes to the host nuclear genome. In the aphid Cinara cedri, the

450 primary endosymbiont Buchnera lacks riboflavin biosynthesis genes, but a more recently integrated

451 Serratia symbiotica associate retains this pathway (Perez-Brocal et al., 2006; Lamelas et al., 2011). In

452 the mealybug Planococcus citri, the symbiont Moranella endobia, which is nested within cells of the

453 endosymbiont Tremblaya princeps, encodes two riboflavin biosynthesis genes. Two other genes are

454 encoded by the host nuclear genome and appear to have been transferred from facultative symbionts

455 during past colonizations (Husnik et al., 2013). In contrast to the above symbioses, our analysis of

456 Illumina read datasets generated from genomic DNA of C. obliquus ants and P. turneri gasters showed

457 no evidence that other bacterial associates or the ant host encode intact riboflavin biosynthesis genes.

458 Rather, the ant hosts may acquire riboflavin via their diet, or it is possible that members of the gut

459 microbiome synthesize this vitamin for the host. Gut bacteria are not represented at high coverage in

460 our read datasets.

461 As with riboflavin, insects lack a pathway for biosynthesis of vitamin B6 (Tanaka, Tateno \&

462 Gojobori, 2005) and thus rely on their diet or symbionts to supply this essential cofactor. Five of the

463 six sequenced Blochmannia encode the 'DXP dependent' pathway for vitamin B6 biosynthesis

464 characteristic of E. coli and other gamma-proteobacteria (Mukherjee et al., 2011) The B. obliquus 
465 lineage has lost all but two of the seven genes in this pathway. In the other Blochmannia lineages, these 466 genes are scattered along the genome rather than adjacent to each other, suggesting loss due to relaxed 467 selective pressure on the pathway instead of a large deletion event affecting multiple genes. This 468 explanation is supported by retention of $d x s$ and $\operatorname{ser} C$ in $B$. obliquus. These genes are also involved in 469 amino acid metabolism and terpenoid backbone biosynthesis pathways, respectively, which are 470 conserved in Blochmannia. The two genes are likely under selective pressure due to their roles in these 471 other metabolic pathways, which prevented their loss in the B. obliquus lineage. In addition to metabolism, Blochmannia lineages show differences in replication and repair genes. Specifically, the chromosomal replication initiation protein $d n a A$ was lost in all but one of the sequenced Blochmannia lineages. Some obligate bacterial endosymbionts of insects, such as Buchnera species, have retained $d n a A$ despite severe genome reduction. By contrast, loss of $d n a A$ has occurred in a few insect endosymbionts, including Baumannia, Blattabacterium, Carsonella, Sulcia and Wigglesworthia (Akman et al., 2002; Nakabachi et al., 2006; Wu et al., 2006; Lopez-Sanchez et al., 2009). DnaA is also missing from a bacterial endosymbiont of protists found in termite guts (Hongoh et al., 2008). Previously, it was hypothesized that loss of $d$ naA was necessary for establishing a stable symbiosis between insects and bacterial endosymbionts located in the cytosol, because it may enable

481 direct control of symbiont DNA replication by the host (Gil et al., 2003). The presence of intact $d n a A$ 482 in B. turneri and a dnaA pseudogene in B. obliquus demonstrates that loss of dnaA was not required for establishment of a stable symbiosis between Blochmannia and camponotines. However, the precise

484 function of DnaA in B. turneri and the mechanisms for controlling initiation of chromosome replication in different Blochmannia lineages are unclear. In addition, it remains untested whether

486 divergent Blochmannia lineages live in the cytosol like B. floridanus or, alternatively, occupy host487 derived vacuoles. 
489 lineages, with either one or the other retained. Loss of $m u t Y$ appears to be more common in 490 intracellular bacteria (Garcia-Gonzalez, Rivera-Rivera \& Massey, 2012). Overexpression of $m u t M$ can

491 "rescue" inactivation of $m u t Y$ in E. coli (Michaels et al., 1992), which implies that retention of mutM 492 may be favored more strongly by selection. However, some bacteria, including obligate intracellular

493 Rickettsia species, lack both mutM and mutY (Garcia-Gonzalez, Rivera-Rivera \& Massey, 2012).

494 Additional sequencing of Blochmannia from diverse ant hosts may reveal the evolutionary trajectories 495 leading to differential loss of mutM and mut $Y$ in Blochmannia.

496 Obligate bacterial endosymbionts of insects are characterized by a high degree of genome 497 stability, with strictly conserved gene order observed among sequenced representatives of some 498 endosymbiont groups, such as Carsonella and Sulcia. Comparative genomics of other endosymbiont 499 genera, including Blattabacterium and Buchnera, have identified typically three or fewer inversions.

500 Recently, an exception to this extreme conservation of genome architecture was described in Portiera, 501 an endosymbiont of whiteflies (Sloan \& Moran, 2013). Analysis of Portiera genomes from divergent 502 whitefly host genera predicted at least 17 inversion events, with most occurring in one lineage that also 503 had a high prevalence of tandem repeats.

Previously, the Blochmannia genome dataset sampled only species from Camponotus hosts, and

505 these four sequenced species shared strictly conserved gene order. By sequencing Blochmannia from

506 ant hosts on divergent branches of the tribe Camponotini, our analysis revealed that inversions have 507 occurred throughout the evolution of this endosymbiont group. Comparisons with close relatives 508 suggest that inversions are not limited to a particular Blochmannia lineage, but rather may have 509 occurred along all four major branches of the phylogeny. We identified gene losses in some of the 510 regions involved in inversion events; it is possible that changes in mutational pressure arising from 511 strand switch contributed to degradation and eventual loss of these genes (Williams \& Wernegreen, 512 2012). 
513 By sequencing genomes from Blochmannia of divergent ant host lineages, we expanded the

514 available Blochmannia genome dataset beyond Camponotus hosts and reconstructed evolutionary

515 trajectories of Blochmannia that likely span the origin of the tribe Camponotini. Analysis of deep

516 branches in symbiont groups addresses questions surrounding the origin of symbioses and the

517 mechanisms involved in establishment of stable associations. Although divergent Blochmannia

518 genomes share much of their gene content, differential gene losses across key functional categories are

519 likely to impact the host-bacterial partnership. It remains challenging to distinguish if different losses

520 reflect selective fine-tuning across distinct ant hosts, stochastic gene deletions or a combination of the

521 two. However, our results, particularly the numerous instances of parallel gene loss, hint that the

522 strength or efficacy of selection to maintain gene functions has varied across ant host lineages and

523 contributed to observed genome variation. These findings contribute to a broader understanding of

524 processes shaping genome reduction in insect endosymbionts and potentially in other bacteria,

525 including intracellular pathogens. 


\section{Acknowledgments}

527 The authors gratefully acknowledge Simon K. A. Robson for providing specimens of P. turneri and 528 Benoit Guénard for providing C. obliquus. We thank Yongliang Fan for preparing genomic DNA and 529 Lisa Bukovnik, Olivier Fedrigo and Fangfei Ye for Illumina sequencing through the core facility in the 530 Center for Genomic and Computational Biology (GCB) at Duke University. We are grateful to Julie Yi 531 for assistance with genome gap closing and analysis of tolA. We thank Michelle G. Giglio at the 532 Institute for Genome Sciences in the University of Maryland School of Medicine for assistance with 533 the MANATEE annotation framework. We thank Philip S. Ward for helpful discussion and for 534 archiving voucher specimens at the Bohart Museum of Entomology, University of California, Davis 535 (UCDC).

538 Funding

539 This work was supported by grants to JJW from the National Institutes of Health (R01GM062626) and 540 the National Science Foundation (MCB-1103113). 


\section{$541 \quad$ Figure Legends}

543 Figure 1. Blochmannia phylogeny. The phylogeny was constructed by Bayesian analysis of 544 concatenated amino acid sequence alignments for 30 genes. The phylogeny is artificially rooted on the 545 branch leading to Hamiltonella. All nodes have $100 \%$ posterior probability. The scale bar shows amino 546 acid substitutions.

548 Figure 2. Gene content of three divergent Blochmannia lineages. The total number of intact genes, 549 including genes that have frameshifts in homopolymer tracts, is shown in bold in each section.

550 Camponotus Bloch LCA includes any intact gene found in at least one of the four sequenced

551 Blochmannia of Camponotus sensu stricto. Note that coaBC is one gene. Although yidC and yidD are 552 separate genes in B. turneri and B. floridanus, these two genes are fused in the other sequenced 553 genomes and therefore counted as one gene here. Similarly, BOBLI757_064 and BOBLI757_065 are 554 counted as one gene because they encode the two domains of bifunctional protein $h l d E$.

556 Figure 3. Blochmannia genome plots. Plots of B. obliquus and B. turneri (sequenced in this study) 557 and B. pennsylvanicus (as a representative of Blochmannia of Camponotus sensu stricto) were 558 constructed with DNAPlotter. Position zero is set to the ATG start of $m n m G$ for each genome. Major 559 and minor tick marks on the outer circle show $100 \mathrm{kbp}$ and $20 \mathrm{kbp}$ increments, respectively. Tracks 1 560 and 2 show CDS in blue on the forward and reverse strands, respectively. Track 3 shows pseudogenes 561 in red. Track 4 shows the eight genomic regions that experienced inversions in at least one of the 562 lineages in grey. These regions are labeled A-H for consistency with Figure 6. Track 5 shows GC skew 563 calculated using 500 bp window size and 50 bp step size. Green shading above the line indicates GC 564 skew greater than the genome average, whereas purple shading below the line indicates GC skew 
smaller than the genome average.

567 Figure 4. Vitamin B6 synthesis encoded by six Blochmannia genomes. The pathway for synthesis of

568 vitamin B6 is annotated with gene distribution in the six sequenced Blochmannia. Camp Bloch refers

569 to all four sequenced Blochmannia of Camponotus sensu stricto, which have the same gene content for

570 this pathway. A plus sign indicates an intact gene, a minus sign indicates a missing gene, and a psi

571 symbol indicates a pseudogene.

573 Figure 5. Parallel gene losses. The distribution of genes lost in multiple independent events (i.e., 574 parallel gene losses) are shown in a phylogenetic context. A plus sign indicates an intact gene, which 575 includes frameshifted genes, a psi symbol indicates a pseudogene and a minus sign indicates that the 576 gene is absent. The gene content of B. chromaiodes and B. pennsylvanicus is identical; therefore, only 577 B. pennsylvanicus is shown here. Acquisition via horizontal gene transfer could, in principle, explain 578 some observed patterns; however, such transfer is unlikely in Blochmannia, and we found no evidence 579 for HGT of genes listed here (see text).

581 Figure 6. Inversions in divergent Blochmannia lineages. Whole genome alignment of $B$.

582 pennsylvanicus (as a representative of Blochmannia of Camponotus sensu stricto), B. turneri and B. 583 obliquus with progressive MAUVE shows inversions as colored blocks below the midline for each 584 genome. The eight genomic regions that experienced inversions are labeled A-H with the number of 585 genes involved in each also shown. 


\section{Supplemental Material}

588 Supplemental Table S1. Pseudogene statistics.

589 To examine gene degradation, we used TBLASTN to align Blochmannia pseudogenes to protein

590 sequences of intact genes from the most closely related lineage.

592 Supplemental Table S2. BLASTN analysis of contigs from de novo assemblies.

594 Supplemental Table S3. Ortholog table.

595 "pan-Camp" includes genes found in at least one of the four Blochmannia of Camponotus sensu stricto.

596 Genomic regions where inversion events occurred in at least one lineage are highlighted in grey. For

597 regions where comparisons to close relatives indicate which lineage likely experienced the inversion

598 event, only that lineage's genes are highlighted. When these comparisons are inconclusive, all three

599 lineages are highlighted. Note that BOBLI757_064 and BOBLI757_065 are domain 1 and domain 2, 600 respectively, of bifunctional protein HldE. 
601 References

602 Abascal F, Zardoya R, Telford MJ. 2010. TranslatorX: multiple alignment of nucleotide

603 sequences guided by amino acid translations. Nucleic Acids Research 38:W7-13.

604

605

606

607

608

609

610

611

612

613

614

615

616

617

618

619

620

621

Abbas CA, Sibirny AA. 2011. Genetic control of biosynthesis and transport of riboflavin and flavin nucleotides and construction of robust biotechnological producers. Microbiology and Molecular Biology Reviews 75:321-360.

Akman L, Yamashita A, Watanabe H, Oshima K, Shiba T, Hattori M, Aksoy S. 2002. Genome sequence of the endocellular obligate symbiont of tsetse flies, Wigglesworthia glossinidia. Nature Genetics 32:402-407.

Altschul SF, Gish W, Miller W, Myers EW, Lipman DJ. 1990. Basic local alignment search tool. Journal of Molecular Biology 215:403-410.

Andersson SGE, Kurland CG. 1998. Reductive evolution of resident genomes. Trends in Microbiology 6:263-268.

Benson G. 1999. Tandem repeats finder: a program to analyze DNA sequences. Nucleic Acids Research 27:573-580.

Brady SG, Schultz TR, Fisher BL, Ward PS. 2006. Evaluating alternative hypotheses for the early evolution and diversification of ants. Proceedings of the National Academy of Sciences of the United States of America 103:18172-18177.

Burge SW, Daub J, Eberhardt R, Tate J, Barquist L, Nawrocki EP, Eddy SR, Gardner PP, Bateman A. 2013. Rfam 11.0: 10 years of RNA families. Nucleic Acids Research 41:D226-232.

Carver T, Thomson N, Bleasby A, Berriman M, Parkhill J. 2009. DNAPlotter: circular and linear interactive genome visualization. Bioinformatics 25:119-120.

Cox MP, Peterson DA, Biggs PJ. 2010. SolexaQA: At-a-glance quality assessment of Illumina secondgeneration sequencing data. BMC Bioinformatics 11:485. 
625 Darling AE, Mau B, Perna NT. 2010. progressiveMauve: multiple genome alignment with gene gain, loss and rearrangement. PLoS One 5:e11147.

627 DePristo MA, Banks E, Poplin R, Garimella KV, Maguire JR, Hartl C, Philippakis AA, del Angel G, 628 Rivas MA, Hanna M, McKenna A, Fennell TJ, Kernytsky AM, Sivachenko AY, Cibulskis K, Gabriel SB, Altshuler D, Daly MJ. 2011. A framework for variation discovery and genotyping using next-generation DNA sequencing data. Nature Genetics 43:491-498.

Douglas AE. 2015. Multiorganismal insects: Diversity and function of resident microorganisms. Annual Review of Entomology 60:2.1-2.18.

633 upgrading for omnivorous carpenter ants by the endosymbiont Blochmannia. BMC Biology 5:48.

Galens K, Orvis J, Daugherty S, Creasy HH, Angiuoli S, White O, Wortman J, Mahurkar A, Giglio MG. 2011. The IGS standard operating procedure for automated prokaryotic annotation. Standards in Genomic Sciences 4:244-251.

Garcia-Gonzalez A, Rivera-Rivera RJ, Massey SE. 2012. The presence of the DNA repair genes mutM, $m u t Y, m u t L$, and $m u t S$ is related to proteome size in bacterial genomes. Frontiers in Genetics 3:3.

Gil R, Silva FJ, Zientz E, Delmotte F, Gonzalez-Candelas F, Latorre A, Rausell C, Kamerbeek J, Gadau J, Holldobler B, van Ham RC, Gross R, Moya A. 2003. The genome sequence of Blochmannia floridanus: comparative analysis of reduced genomes. Proceedings of the National Academy of Sciences of the United States of America 100:9388-9393.

Godlewska R, Wisniewska K, Pietras Z, Jagusztyn-Krynicka EK. 2009. Peptidoglycan-associated lipoprotein (Pal) of Gram-negative bacteria: function, structure, role in pathogenesis and potential application in immunoprophylaxis. FEMS Microbiology Letters 298:1-11. 
649 Gordon D, Abajian C, Green P. 1998. Consed: a graphical tool for sequence finishing. Genome $650 \quad$ Research 8:195-202.

651 Hansen FG, Christensen BB, Nielsen CB, Atlung T. 2006. Insights into the quality of DnaA boxes and 652 their cooperativity. Journal of Molecular Biology 355:85-95.

653 Herbeck JT, Degnan PH, Wernegreen JJ. 2005. Nonhomogeneous model of sequence evolution 654 indicates independent origins of primary endosymbionts within the Enterobacteriales (gammaproteobacteria). Molecular Biology and Evolution 22:520-532.

Hongoh Y, Sharma VK, Prakash T, Noda S, Taylor TD, Kudo T, Sakaki Y, Toyoda A, Hattori M, Ohkuma M. 2008. Complete genome of the uncultured Termite Group 1 bacteria in a single host protist cell. Proceedings of the National Academy of Sciences of the United States of America 105:5555-5560.

Husnik F, Chrudimsky T, Hypsa V. 2011. Multiple origins of endosymbiosis within the

\section{3}

664

665

666

667

668

669 Enterobacteriaceae (gamma-proteobacteria): convergence of complex phylogenetic approaches. BMC Biology 9:87.

Husnik F, Nikoh N, Koga R, Ross L, Duncan RP, Fujie M, Tanaka M, Satoh N, Bachtrog D, Wilson AC, von Dohlen CD, Fukatsu T, McCutcheon JP. 2013. Horizontal gene transfer from diverse bacteria to an insect genome enables a tripartite nested mealybug symbiosis. Cell 153:15671578.

Katoh K, Kuma K, Toh H, Miyata T. 2005. MAFFT version 5: improvement in accuracy of multiple sequence alignment. Nucleic Acids Research 33:511-518.

Kikuchi Y. 2009. Endosymbiotic bacteria in insects: their diversity and culturability. Microbes and Environments 24:195-204.

Koboldt DC, Chen K, Wylie T, Larson DE, McLellan MD, Mardis ER, Weinstock GM, Wilson RK, Ding L. 2009. VarScan: variant detection in massively parallel sequencing of individual and 
pooled samples. Bioinformatics 25:2283-2285.

674 Lamelas A, Gosalbes MJ, Manzano-Marin A, Pereto J, Moya A, Latorre A. 2011. Serratia symbiotica 675 from the aphid Cinara cedri: a missing link from facultative to obligate insect endosymbiont. $676 \quad$ PLoS Genetics 7:e1002357.

677 Lee WP, Stromberg MP, Ward A, Stewart C, Garrison EP, Marth GT. 2014. MOSAIK: a hash-based 678 algorithm for accurate next-generation sequencing short-read mapping. PLoS One 9:e90581.

679 Li H, Durbin R. 2009. Fast and accurate short read alignment with Burrows-Wheeler transform. $680 \quad$ Bioinformatics 25:1754-1760.

681 Lind PA, Andersson DI. 2008. Whole-genome mutational biases in bacteria. Proceedings of the 682 National Academy of Sciences of the United States of America 105:17878-17883.

683 Lopez-Sanchez MJ, Neef A, Pereto J, Patino-Navarrete R, Pignatelli M, Latorre A, Moya A. 2009. 684 Evolutionary convergence and nitrogen metabolism in Blattabacterium strain Bge, primary 685 endosymbiont of the cockroach Blattella germanica. PLoS Genetics 5:e1000721.

686 Mackiewicz P, Zakrzewska-Czerwinska J, Zawilak A, Dudek MR, Cebrat S. 2004. Where does 687 688 bacterial replication start? Rules for predicting the oriC region. Nucleic Acids Research $32: 3781-3791$.

689 Michaels ML, Cruz C, Grollman AP, Miller JH. 1992. Evidence that MutY and MutM combine to 690 prevent mutations by an oxidatively damaged form of guanine in DNA. Proceedings of the

692 Moran NA, McCutcheon JP, Nakabachi A. 2008. Genomics and evolution of heritable bacterial 693 symbionts. Annual Review of Genetics 42:165-190.

694 Moreau CS, Bell CD. 2013. Testing the museum versus cradle tropical biological diversity hypothesis: 695 phylogeny, diversification, and ancestral biogeographic range evolution of the ants. Evolution 696 $67: 2240-2257$. 
697 Moya A, Pereto J, Gil R, Latorre A. 2008. Learning how to live together: genomic insights into prokaryote-animal symbioses. Nature Reviews: Genetics 9:218-229.

699 Mrazek J, Xie S. 2006. Pattern locator: a new tool for finding local sequence patterns in genomic DNA sequences. Bioinformatics 22:3099-3100.

701 Mukherjee T, Hanes J, Tews I, Ealick SE, Begley TP. 2011. Pyridoxal phosphate: biosynthesis and catabolism. Biochimica et Biophysica Acta: Protein Structure and Molecular Enzymology 1814:1585-1596.

704 705 endosymbiotic bacteria, Buchnera. Journal of Insect Physiology 45:1-6.

Nakabachi A, Yamashita A, Toh H, Ishikawa H, Dunbar HE, Moran NA, Hattori M. 2006. The 160kilobase genome of the bacterial endosymbiont Carsonella. Science 314:267.

Patino-Navarrete R, Moya A, Latorre A, Pereto J. 2013. Comparative genomics of Blattabacterium cuenoti: the frozen legacy of an ancient endosymbiont genome. Genome Biology and Evolution $5: 351-361$

Perez-Brocal V, Gil R, Ramos S, Lamelas A, Postigo M, Michelena JM, Silva FJ, Moya A, Latorre A. 2006. A small microbial genome: the end of a long symbiotic relationship? Science 314:312313.

Ronquist F, Huelsenbeck JP. 2003. MrBayes 3: Bayesian phylogenetic inference under mixed models. Bioinformatics 19:1572-1574.

Russell JA, Funaro CF, Giraldo YM, Goldman-Huertas B, Suh D, Kronauer DJ, Moreau CS, Pierce NE. 2012. A veritable menagerie of heritable bacteria from ants, butterflies, and beyond: broad molecular surveys and a systematic review. PLoS One 7:e51027.

Sabree ZL, Kambhampati S, Moran NA. 2009. Nitrogen recycling and nutritional provisioning by Blattabacterium, the cockroach endosymbiont. Proceedings of the National Academy of 
Sciences of the United States of America 106:19521-19526.

722 Sameshima S, Hasegawa E, Kitade O, Minaka N, Matsumoto T. 1999. Phylogenetic comparison of

723

724

725

726

727

728

729

730

731

732

733

734

735

736

737

738

739

740

741

742

743

744 endosymbionts with their host ants based on molecular evidence. Zoological Science 16:9931000.

Sauer C, Stackebrandt E, Gadau J, Holldobler B, Gross R. 2000. Systematic relationships and cospeciation of bacterial endosymbionts and their carpenter ant host species: proposal of the new taxon Candidatus Blochmannia gen. nov. International Journal of Systematic and Evolutionary Microbiology 50 Pt 5:1877-1886.

Schaper S, Messer W. 1995. Interaction of the initiator protein DnaA of Escherichia coli with Its DNA target. Journal of Biological Chemistry 270:17622-17626.

Shigenobu S, Watanabe H, Hattori M, Sakaki Y, Ishikawa H. 2000. Genome sequence of the endocellular bacterial symbiont of aphids Buchnera sp. APS. Nature 407:81-86.

Sloan DB, Moran NA. 2013. The evolution of genomic instability in the obligate endosymbionts of whiteflies. Genome Biology and Evolution 5:783-793.

Stoll S, Feldhaar H, Fraunholz MJ, Gross R. 2010. Bacteriocyte dynamics during development of a holometabolous insect, the carpenter ant Camponotus floridanus. BMC Microbiology 10:308.

Tamas I, Wernegreen JJ, Nystedt B, Kauppinen SN, Darby AC, Gomez-Valero L, Lundin D, Poole AM, Andersson SG. 2008. Endosymbiont gene functions impaired and rescued by polymerase infidelity at poly(A) tracts. Proceedings of the National Academy of Sciences of the United States of America 105:14934-14939.

Tanaka T, Tateno Y, Gojobori T. 2005. Evolution of vitamin B6 (pyridoxine) metabolism by gain and loss of genes. Molecular Biology and Evolution 22:243-250.

Wall DP, Fraser HB, Hirsh AE. 2003. Detecting putative orthologs. Bioinformatics 19:1710-1711.

Wernegreen JJ, Kauppinen SN, Brady SG, Ward PS. 2009. One nutritional symbiosis begat another: 

phylogenetic evidence that the ant tribe Camponotini acquired Blochmannia by tending sapfeeding insects. BMC Evolutionary Biology 9:292.

Wernegreen JJ, Kauppinen SN, Degnan PH. 2010. Slip into something more functional: selection maintains ancient frameshifts in homopolymeric sequences. Molecular Biology and Evolution 27:833-839.

Werren JH, Baldo L, Clark ME. 2008. Wolbachia: master manipulators of invertebrate biology. Nature Reviews: Microbiology 6:741-751.

Williams LE, Wernegreen JJ. 2010. Unprecedented loss of ammonia assimilation capability in a urease-encoding bacterial mutualist. BMC Genomics 11:687.

Williams LE, Wernegreen JJ. 2012. Purifying selection, sequence composition, and context-specific indel mutations shape intraspecific variation in a bacterial endosymbiont. Genome Biology and Evolution 4:44-51.

Williams LE, Wernegreen JJ. 2013. Sequence context of indel mutations and their effect on protein evolution in a bacterial endosymbiont. Genome Biology and Evolution 5:599-605.

Wu D, Daugherty SC, Van Aken SE, Pai GH, Watkins KL, Khouri H, Tallon LJ, Zaborsky JM, Dunbar HE, Tran PL, Moran NA, Eisen JA. 2006. Metabolic complementarity and genomics of the dual bacterial symbiosis of sharpshooters. PLoS Biology 4:e188.

Wu M, Chatterji S, Eisen JA. 2012. Accounting for alignment uncertainty in phylogenomics. PLoS One 7:e30288.

Yang Y, Zhao G, Man T-K, Winkler ME. 1998. Involvement of the gapA- and epd (gapB)-encoded dehydrogenases in pyridoxal 5'-phosphate coenzyme biosynthesis in Escherichia coli K-12. Journal of Bacteriology 180:4294-4299.

Zakrzewska-Czerwinska J, Jakimowicz D, Zawilak-Pawlik A, Messer W. 2007. Regulation of the initiation of chromosomal replication in bacteria. FEMS Microbiology Reviews 31:378-387. 
769 Zerbino DR, Birney E. 2008. Velvet: algorithms for de novo short read assembly using de Bruijn $770 \quad$ graphs. Genome Research 18:821-829.

771 Zhou K, Vanoirbeek K, Aertsen A, Michiels CW. 2012. Variability of the tandem repeat region of the $772 \quad$ Escherichia coli tolA gene. Research in Microbiology 163:316-322.

773 Zientz E, Beyaert I, Gross R, Feldhaar H. 2006. Relevance of the endosymbiosis of Blochmannia 774 floridanus and carpenter ants at different stages of the life cycle of the host. Applied and $775 \quad$ Environmental Microbiology 72:6027-6033. 


\section{Table $\mathbf{1}$ (on next page)}

Genome statistics of six sequenced Blochmannia. 
Table 1. Genome statistics of six sequenced Blochmannia.

\begin{tabular}{|c|c|c|c|c|c|c|c|c|c|}
\hline Genome & Size & $\begin{array}{c}\text { GC } \\
\text { content } \\
(\%)\end{array}$ & $\begin{array}{c}\text { Genes } \\
\text { (inc. } \\
\text { pseudo) }\end{array}$ & $\begin{array}{c}\text { Protein } \\
\text { coding }\end{array}$ & tRNA & rRNA & $\begin{array}{c}\text { other } \\
\text { RNA }\end{array}$ & $\begin{array}{c}\text { pseudo } \\
\text { genes }\end{array}$ & $\begin{array}{c}\text { frameshifted } \\
\text { genes }\end{array}$ \\
\hline B. obliquus & 773,940 & 27.4 & 642 & 584 & 37 & 3 & 3 & 15 & 4 \\
\hline B. turneri & 749,321 & 29.1 & 634 & 589 & 38 & 3 & 3 & 1 & 7 \\
\hline B. chromaiodes & 791,219 & 29.5 & 658 & 609 & 40 & 3 & 3 & 3 & 4 \\
\hline B. pennsylvanicus & 791,654 & 29.6 & 658 & 609 & 40 & 3 & 3 & 3 & 4 \\
\hline B. floridanus & 705,557 & 27.4 & 637 & 590 & 37 & 3 & 3 & 4 & 4 \\
\hline B. vafer & 722,585 & 27.5 & 631 & 587 & 37 & 3 & 2 & 2 & 8 \\
\hline
\end{tabular}


1

Blochmannia phylogeny.

The phylogeny was constructed by Bayesian analysis of concatenated amino acid sequence alignments for 30 genes. The phylogeny is artificially rooted on the branch leading to Hamiltonella. All nodes have $100 \%$ posterior probability. The scale bar shows amino acid substitutions.

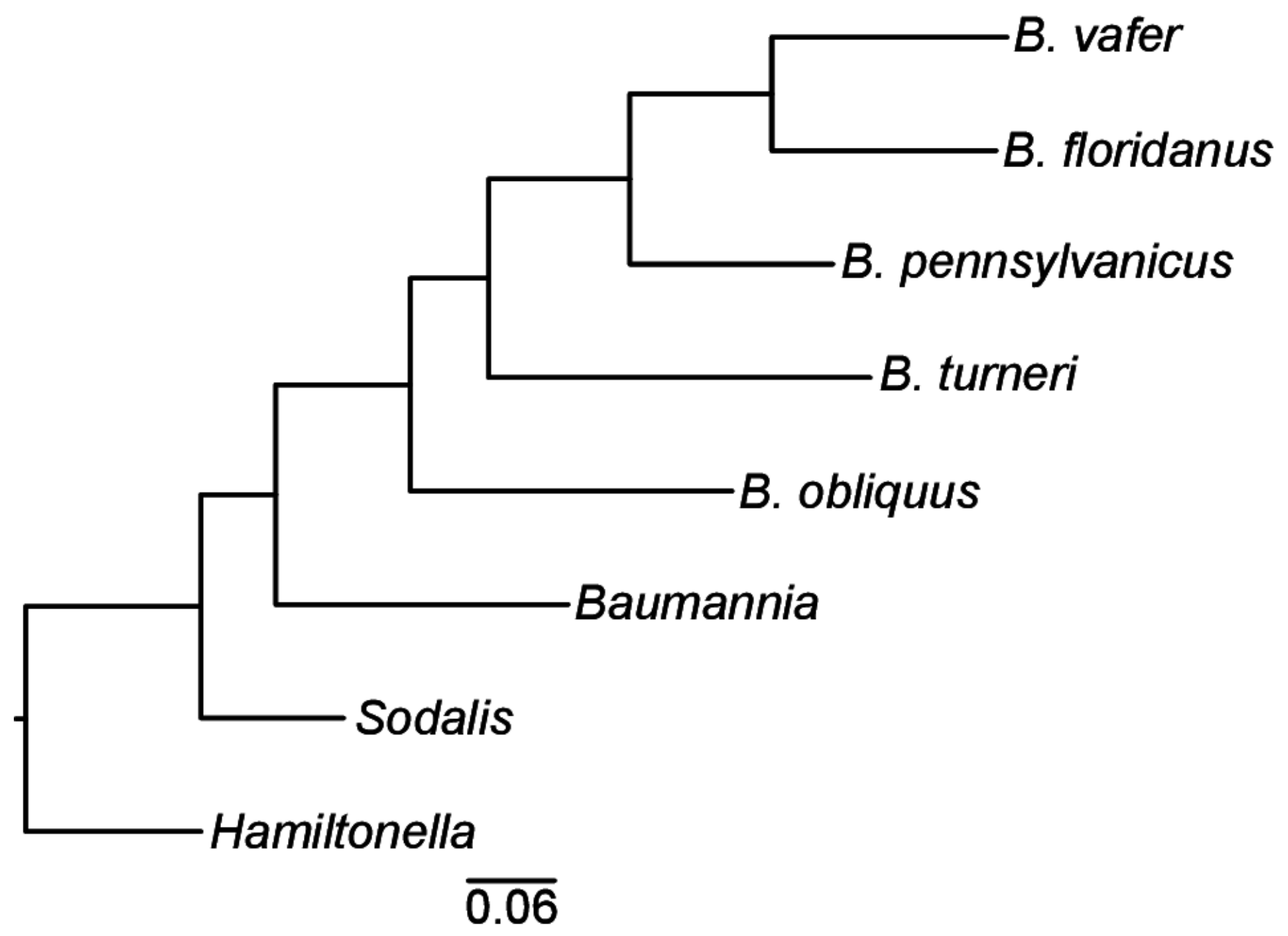




\section{2}

Gene content of three divergent Blochmannia lineages.

The total number of intact genes, including genes that have frameshifts in homopolymer tracts, is shown in bold in each section. Camponotus Bloch LCA includes any intact gene found in at least one of the four sequenced Blochmannia of Camponotus sensu stricto. Note that $c o a B C$ is one gene. Although $y i d C$ and $y i d D$ are separate genes in $B$. turneri and $B$. floridanus, these two genes are fused in the other sequenced genomes and therefore counted as one gene here. Similarly, BOBLI757_064 and BOBLI757_065 are counted as one gene because they encode the two domains of bifunctional protein $h / d E$. 


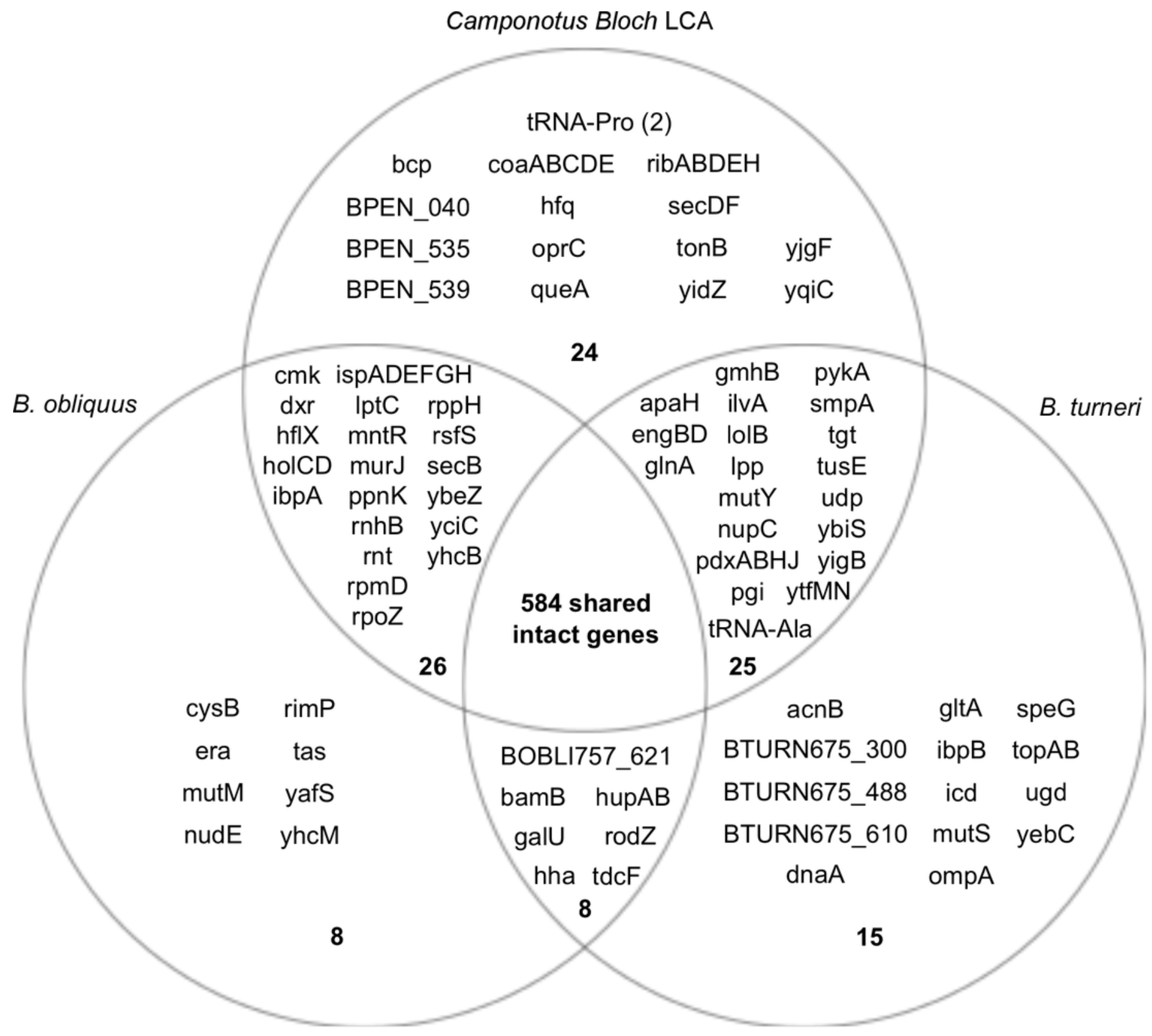




\section{Figure $\mathbf{3}$ (on next page)}

\section{Blochmannia genome plots}

Plots of $B$. obliquus and $B$. turneri (sequenced in this study) and $B$. pennsylvanicus (as a representative of Blochmannia of Camponotus sensu stricto) were constructed with DNAPlotter. Position zero is set to the ATG start of $m n m G$ for each genome. Major and minor tick marks on the outer circle show $100 \mathrm{kbp}$ and $20 \mathrm{kbp}$ increments, respectively. Tracks 1 and 2 show CDS in blue on the forward and reverse strands, respectively. Track 3 shows pseudogenes in red. Track 4 shows the eight genomic regions that experienced inversions in at least one of the lineages in grey. These regions are labeled $\mathrm{A}-\mathrm{H}$ for consistency with Figure 6. Track 5 shows GC skew calculated using 500 bp window size and 50 bp step size. Green shading above the line indicates GC skew greater than the genome average, whereas purple shading below the line indicates GC skew smaller than the genome average. 
4

Vitamin B6 synthesis encoded by six Blochmannia genomes.

The pathway for synthesis of vitamin B6 is annotated with gene distribution in the six sequenced Blochmannia. Camp Bloch refers to all four sequenced Blochmannia of Camponotus sensu stricto, which have the same gene content for this pathway. A plus sign indicates an intact gene, a minus sign indicates a missing gene, and a psi symbol indicates a pseudogene. 


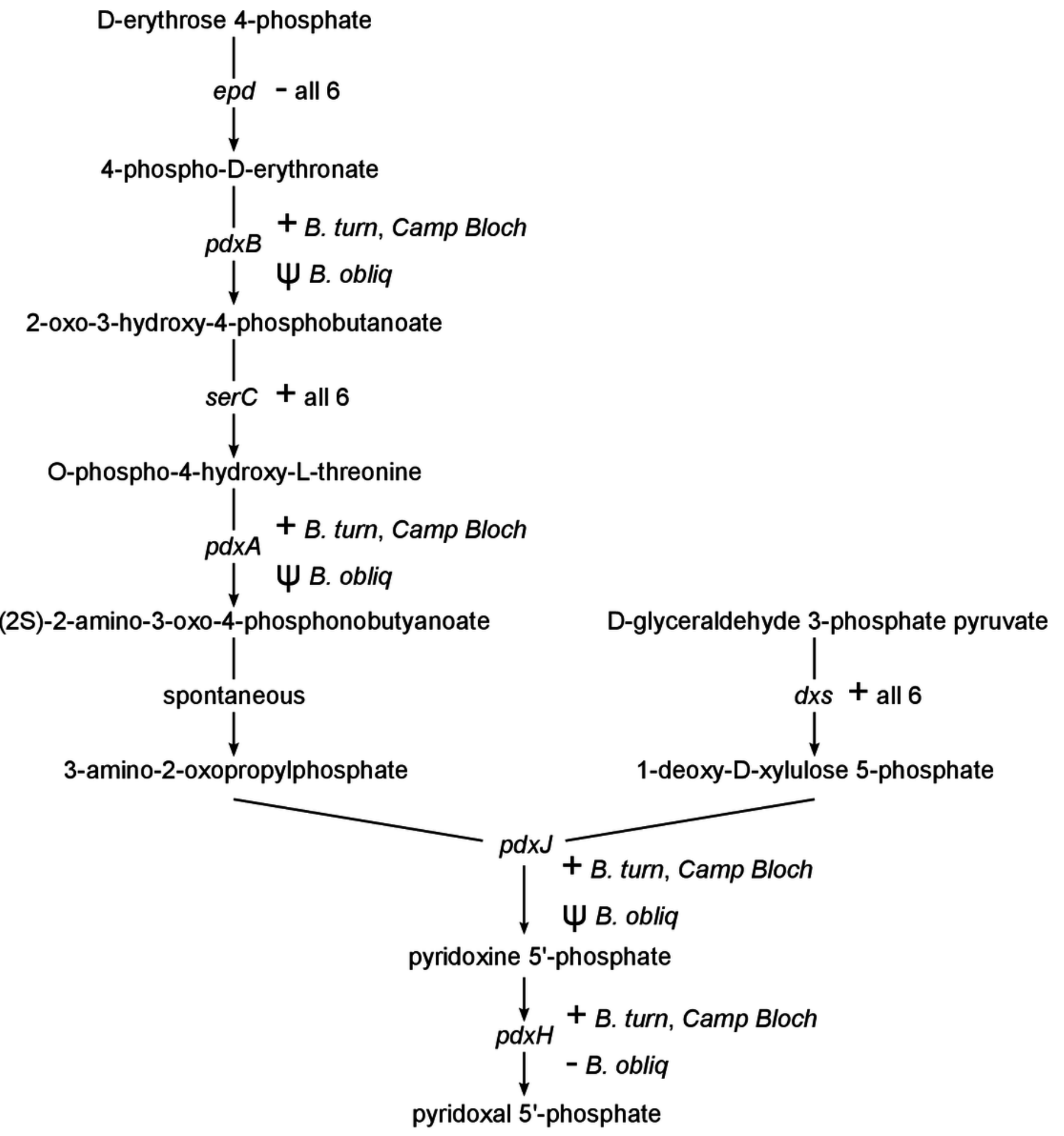




\section{Table 2(on next page)}

Tandem repeats within tolA of Blochmannia and E. coli.

Data from Tandem Repeats Finder (Benson, 1999).* Four repeat patterns were identified. The top-scoring pattern is reported here. 
Table 2. Tandem repeats within tolA of Blochmannia and E. coli.

\begin{tabular}{|c|c|c|c|c|c|}
\hline Genome & $\begin{array}{c}\text { Length of tolA } \\
\text { (bp) }\end{array}$ & $\begin{array}{c}\text { Length of } \\
\text { consensus } \\
\text { repeat pattern } \\
\text { (bp) }\end{array}$ & $\begin{array}{c}\text { \# of repeat } \\
\text { copies }\end{array}$ & Alignment score & $\begin{array}{c}\text { Coordinates of } \\
\text { repeat region }\end{array}$ \\
\hline B. obliquus & 771 & 17 & 2.1 & 52 & $81-114$ \\
\hline B. turneri & 990 & 81 & 4.4 & 699 & $205-558$ \\
\hline B. chromaiodes & 1185 & 87 & 6.0 & 1030 & $199-722$ \\
\hline B. pennsylvanicus & 1185 & 87 & 6.0 & 1046 & $200-722$ \\
\hline B. floridanus & 1305 & 99 & 6.5 & 1260 & $215-853$ \\
\hline B. vafer & 1173 & 102 & 5.1 & 1038 & $208-726$ \\
\hline E. coli MG1655* & 1266 & 79 & 2.0 & 239 & $671-829$ \\
\hline
\end{tabular}

Data from Tandem Repeats Finder (Benson, 1999)

*Four repeat patterns were identified. The top-scoring pattern is reported here. 


\section{5}

Parallel gene losses.

The distribution of genes lost in multiple independent events (i.e., parallel gene losses) are shown in a phylogenetic context. A plus sign indicates an intact gene, which includes frameshifted genes, a psi symbol indicates a pseudogene and a minus sign indicates that the gene is absent. The gene content of $B$. chromaiodes and B. pennsylvanicus is identical; therefore, only $B$. pennsylvanicus is shown here. Acquisition via horizontal gene transfer could, in principle, explain some observed patterns; however, such transfer is unlikely in Blochmannia, and we found no evidence for HGT of genes listed here (see text).
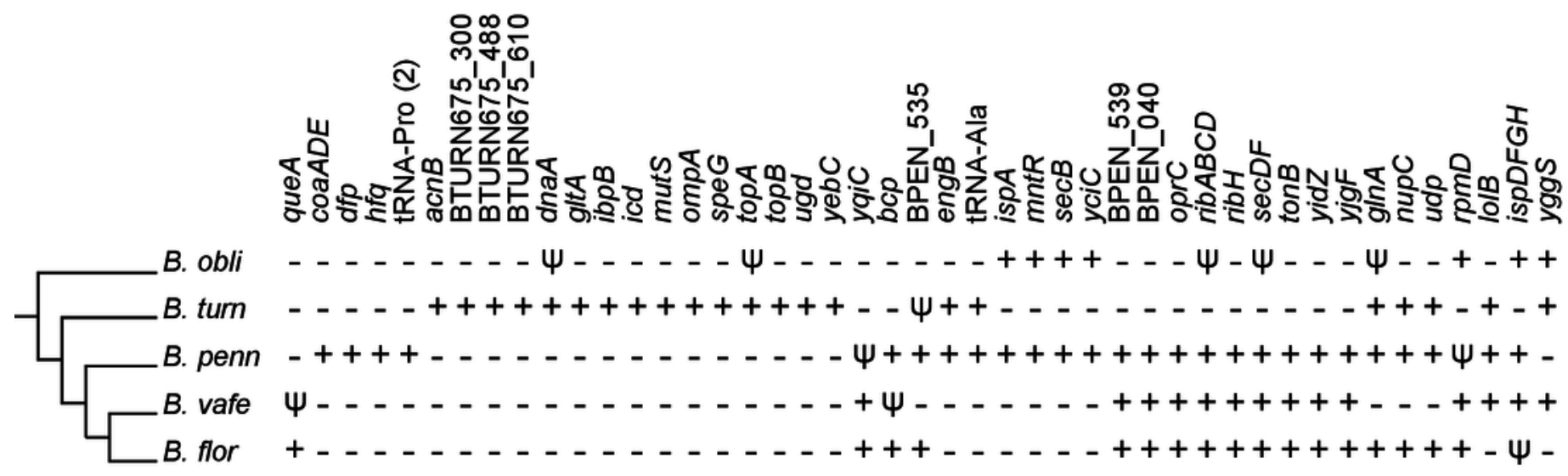
Figure $\mathbf{6}$ (on next page)

Inversions in divergent Blochmannia lineages.

Whole genome alignment of $B$. pennsylvanicus (as a representative of Blochmannia of Camponotus sensu stricto), $B$. turneri and $B$. obliquus with progressive MAUVE shows inversions as colored blocks below the midline for each genome. The eight genomic regions that experienced inversions are labeled A-H with the number of genes involved in each also shown. 
